\title{
CHERENKOV RADIATION OF THE RELATIVISTIC ELECTRON BUNCH IN ION DIELECTRIC MEDIA
}

\author{
V.A. Balakirev, I.N. Onishchenko \\ National Science Center “Kharkov Institute of Physics and Technology”, Kharkiv, Ukraine \\ E-mail:onish@kipt.kharkov.ua
}

The process of wakefields excitation by the relativistic electron bunch in dielectric media with ion type of chemical bond is studied. The spatio-temporal structure of the excited wakefield in ion dielectric waveguide is obtained and investigated. It is shown that the excited wakefield in the infrared and longer wavelength ranges consists of the field of longitudinal optical phonons and Cherenkov radiation as a set of eigen electromagnetic waves of the dielectric waveguide.

PACS: 41.75.Lx, 41.85.Ja, 41.69.Bq

\section{INTRODUCTION}

The Cherenkov radiation effect of charged particles (bunches of charged particles) moving in a dielectric medium can be used for realization of the wakefield method of charged particles acceleration [1 - 3]. Moreover, as a rule, the wakefield excitation process was considered without taking into account the frequency dispersion of the dielectric constant of the medium $\varepsilon(\omega)=\varepsilon_{0}=$ Const . Meanwhile, taking into account the dependence of the dielectric constant on frequency leads to a number of qualitative features of the picture of wakefield excitation in dielectric structures. First of all, it is possible to excite longitudinal potential oscillations of a crystal dielectric, for example, longitudinal optical phonons in dielectric crystal media with ion bond. In addition, in an ion dielectric in the infrared frequency range there is an additional branch of transverse electromagnetic waves. The Cherenkov excitation of electromagnetic waves belonging to this branch is also of interest.

In the present work, the process of excitation of wake electromagnetic fields in an ion dielectric medium by a relativistic electron bunch (REB) is investigated. For definiteness, we will consider ion alkali-halide dielectrics with the formula $A_{I} B_{V I I}$, where $A_{I}$ is any element of the periodic table belonging to the first group (alkali metals, for example, $\mathrm{Na}, \mathrm{K}$, etc.), $B_{V I I}$ is any seventh group halide element, for example $\mathrm{F}, \mathrm{Cl}$, etc. The choice of alkaline-halide crystal dielectrics is primarily due to their relatively simple internal structure and, accordingly, a simple dependence $\varepsilon(\omega)$, which allows us to study the process of wakefields excitation by REB with analytical methods. Our aim is to study the frequency spectrum of excited wakefields and their spatiotemporal structures.

We restrict ourselves to the study of wake fields in the infrared and lower frequency ranges. This is due to the fact that for efficient excitation of the wavefield by an electron bunch, it is necessary to achieve coherence of electromagnetic waves excitation. For this, it is necessary that the longitudinal and transverse dimensions of the electron bunch should be less (substantially less) than the length of the excited wave. For optical and especially ultraviolet frequency ranges, this requirement is very problematic. And if this requirement is not satisfied, the amplitude of the wake wave will be insignificant.

\section{STATEMENT OF THE PROBLEM. BASIC EQUATIONS}

Let's consider the homogeneous dielectric cylinder of radius $b$, the side surface of which is covered with a perfectly conductive metal film. Along the axis of the dielectric waveguide, an axisymmetric REB moves uniformly and rectilinearly. The initial system of equations contains Maxwell's equations

$$
\begin{gathered}
\operatorname{rot} \vec{E}=-\frac{1}{c} \frac{\partial \vec{H}}{\partial t}, \quad \operatorname{rot} \vec{H}=\frac{1}{c} \frac{\partial \vec{D}}{\partial t}+\frac{4 \pi}{c} \vec{j}_{b} \\
\operatorname{div} \vec{D}=4 \pi \rho, \quad \operatorname{div} \vec{H}=0,
\end{gathered}
$$

$\rho_{b}, \vec{j}_{b}$ are charge density and current of an electron bunch, $\vec{D}=\hat{\varepsilon} \vec{E}$ is electric displacement field, $\hat{\varepsilon}$ is dielectric constant operator of an ion dielectric.

The system of Maxwell equations (1) describes the excitation of an electromagnetic field by external charges and currents in a condensed dielectric medium.

\section{DETERMINATION OF THE GREEN FUNCTION}

We will solve the problem of wake field excitation by an axisymmetric electron bunch in a dielectric waveguide as follows. First, we determine the field (Green's function) of a moving charge in the form of an infinitely thin ring with a charge density

$$
d \rho=-d Q \frac{1}{v_{0}} \frac{\delta\left(r-r_{0}\right)}{2 \pi r_{0}} \delta\left(t-\frac{z}{v_{0}}-t_{0}\right),
$$

where $r$ is radial coordinate, $r_{0}$ is ring radius, $t_{0}$ is time of entry of an elementary ring bunch into the waveguide, $v_{0}$ is bunch velocity, $d Q\left(r_{0}, t_{0}\right)$ is the elementary charge of the ring connected with the current density of the bunch at the entrance to the dielectric waveguide $(z=0) \quad j_{0}\left(t_{0}, r_{0}\right)$ by the relation

$$
d Q=j_{0}\left(t_{0}, r_{0}\right) 2 \pi r_{0} d r_{0} d t_{0} .
$$

The current density of an elementary ring charge is determined by the expression

$$
\overrightarrow{d j}=v_{0} d \rho \vec{e}_{z},
$$

$\vec{e}_{z}$ is unit vector in longitudinal direction.

Let's consider a bunch of electrons with the current density

$$
j_{0}\left(r_{0}, t_{0}\right)=j_{0} R\left(r_{0} / r_{b}\right) T\left(t_{0} / t_{b}\right),
$$


where the function $R\left(r_{0} / r_{b}\right)$ describes dependence of the bunch density on radius (transverse profile), $r_{b}$ is characteristic transverse bunch size, function $T\left(t_{0} / t_{b}\right)$ describes the longitudinal density profile of a bunch, $t_{b}$ is characteristic duration of the bunch. The value $j_{0}$ is connected with a full charge $Q$ by the relation $j_{0}=Q /\left(s_{\text {eff }} t_{\text {eff }}\right)$, where $s_{\text {eff }}$ is effective cross section of the bunch

$$
s_{\text {eff }}=\pi r_{b}^{2} \hat{\sigma}, \hat{\sigma}=2 \int_{0}^{\infty} R(\rho) \rho d \rho,
$$

and $t_{\text {eff }}$ is effective bunch duration

$$
t_{\text {eff }}=\hat{\tau} t_{b} . \hat{\tau}=\int_{-\infty}^{\infty} T\left(\tau_{0}\right) d \tau_{0} .
$$

We expand the quantities in the Maxwell equations (1) into the Fourier integrals over frequencies

$$
\begin{gathered}
\left(\vec{E}_{G}, \vec{H}_{G}\right)=\int_{-\infty}^{\infty}\left(\vec{E}_{\omega}, \vec{H}_{\omega}\right) e^{-i \omega \tau} d \omega \\
\left(\vec{j}_{b}, d \rho_{b}\right)=-d Q \frac{\delta\left(r-r_{0}\right)}{2 \pi r_{0}} \frac{1}{2 \pi} \int_{-\infty}^{\infty}\left(\vec{e}_{z}, \frac{1}{v_{0}}\right) e^{-i \omega \tau} d \omega,
\end{gathered}
$$

$\tau=t-z / v_{0}-t_{0}, \quad \vec{E}_{G}, \vec{H}_{G} \quad$ is electromagnetic field (Green's function) of elementary current and charge (6).

The system of Maxwell equations (1), taking into account relations (5), (6), can be transformed to the equation for the longitudinal Fourier component of the electric field

$$
\begin{gathered}
\frac{1}{r} \frac{d}{d r} r \frac{d E_{G z \omega}}{d r}+k_{\perp}^{2} E_{G z \omega}=\frac{i}{\pi} \frac{k_{\perp}^{2}}{\omega \varepsilon(\omega)} d Q \frac{\delta\left(r-r_{0}\right)}{r_{0}}, \\
k_{\perp}^{2}=k_{0}^{2} \varepsilon(\omega)-k^{2}, k=\omega / v_{0}, k_{0}=\omega / c, \varepsilon(\omega)
\end{gathered}
$$

is dielectric constant of an ion dielectric. On the perfectly conducting side surface of the dielectric waveguide $r=b$, the longitudinal component of the electric field vanishes

$$
E_{z \omega}(r=b)=0 \text {. }
$$

We will search the longitudinal Fourier component of the electric field $E_{G z \omega}$ in the form of a series of Bessel functions

$$
E_{G z \omega}=\sum_{n=1}^{\infty} C_{n}(\omega) J_{0}\left(\lambda_{n} r / b\right),
$$

where $\lambda_{n}$ are the roots of the Bessel function $J_{0}(x)$. Using the orthogonality of the Bessel functions $J_{0}\left(\lambda_{n} r / b\right)$, from equation (7) we find the expansion coefficients

$$
C_{n}(\omega)=\frac{i}{\pi} d Q \frac{i}{\omega \varepsilon(\omega)} \frac{k_{\perp}^{2}}{k_{\perp}^{2}-\frac{\lambda_{n}^{2}}{b^{2}}} \frac{J_{0}\left(\lambda_{n} r_{0} / b\right) J_{0}\left(\lambda_{n} r / b\right)}{N_{n}}
$$

Here $N_{n}=\frac{b^{2}}{2} J_{1}^{2}\left(\lambda_{n}\right)$ is wave norm. Accordingly, for the longitudinal component of the electric field we have the following expression

$$
E_{G z}(r, \tau)=\frac{i}{\pi} d Q \sum_{n=1}^{\infty} \frac{J_{0}\left(\lambda_{n} r_{0} / b\right) J_{0}\left(\lambda_{n} r / b\right)}{N_{n}} S_{n}(\tau),
$$

where

$$
\begin{gathered}
S_{n}(\tau)=\int_{-\infty}^{\infty} e^{-i \omega \tau} \frac{d \omega}{\omega} \frac{k_{\perp}^{2}(\omega)}{\varepsilon(\omega) D_{n}(\omega)}, \\
D_{n}(\omega)=k_{0}^{2} \varepsilon(\omega)-k^{2}-\frac{\lambda_{n}^{2}}{b^{2}} .
\end{gathered}
$$

The dielectric constant of an ion alkali - halide dielectric can be represented in the form [4]

$$
\varepsilon(\omega)=\frac{\left(\omega^{2}-\omega_{L i}^{2}\right)\left(\omega^{2}-\omega_{L e-}^{2}\right)\left(\omega^{2}-\omega_{L e++}^{2}\right)}{\left(\omega^{2}-\omega_{T i}^{2}\right)\left(\omega^{2}-\omega_{T e-}^{2}\right)\left(\omega^{2}-\omega_{T e+}^{2}\right)} .
$$

The characteristic frequencies in relation (13) are arranged in magnitude as follows

$$
\omega_{T i}<\omega_{L i}<\omega_{T e-}<\omega_{L e-}<\omega_{T e+}<\omega_{L e+}
$$

Frequencies $\omega_{L i}, \omega_{L e( \pm)}$ are zeros of dielectric permittivity $\varepsilon(\omega)=0$. Frequency $\omega_{L i}$ is the frequency of longitudinal optical phonons and lies in the infrared frequency range. Frequencies $\omega_{L e( \pm)}$ are the frequencies of longitudinal polarized electron oscillations and lie in the optical or even ultraviolet frequency range. We also note that the frequencies $\omega_{L i}, \omega_{L e( \pm)}$ are also cutoff frequencies for normal incidence of electromagnetic waves on a plane dielectric layer. In turn, the frequencies $\omega_{T i}, \omega_{T e( \pm)}$ are the poles of the dielectric constant and determine the absorption lines of the electromagnetic waves of the ion crystal. In the vicinity of these frequencies, the imaginary part of the dielectric constant and, accordingly, the energy loss of electromagnetic waves increases abnormally.

The absorption frequency of ion subsystems $\omega_{T i}$ is the frequency of transverse optical phonons. We note that the optical longitudinal and transverse acoustic branches of the oscillations are characterized by the fact that in the unit cell of the ion crystal, oppositely charged ions are shifted towards each other. In this case, the center of mass of the unit cell remains motionless. As in the case of longitudinal optical phonons, the frequencies of transverse optical phonons lie in the infrared range. Electronic resonance absorption frequencies are in the optical range.

For the infrared range, the expression for the dielectric constant (13) takes the form [5 - 7]

$$
\varepsilon(\omega)=\varepsilon_{\text {opt }} \frac{\left(\omega^{2}-\omega_{L e+}^{2}\right)}{\left(\omega^{2}-\omega_{T e+}^{2}\right)},
$$

where

$$
\varepsilon_{\text {opt }}=\frac{\omega_{L e-}^{2}}{\omega_{T e-}^{2}} \frac{\omega_{L e+}^{2}}{\omega_{T e+}^{2}},
$$

$\varepsilon_{\text {opt }}$ is optical dielectric permittivity.

The zeros of the dielectric constant are the poles of the integrand (12). Calculating the residues at the poles $\omega= \pm \omega_{L i}-i 0$, we find the potential part of the Green's function

$$
\begin{gathered}
E_{G z}^{(l)}(r, \tau)=2 d Q \frac{\omega_{L i}^{2}}{v_{0}^{2} \varepsilon_{e f f}} G\left(k_{L i} r, k_{L i} r_{0}\right) \vartheta(\tau) \cos \omega_{L i} \tau, \\
k_{L i}=\omega_{L i} / v_{0}, \varepsilon_{e f f}=\frac{\varepsilon_{s t} \varepsilon_{o p t}}{\Delta \varepsilon}, \varepsilon_{s t}=\varepsilon_{o p t} \frac{\omega_{L i}^{2}}{\omega_{T i}^{2}},
\end{gathered}
$$

$\varepsilon_{s t}$ is static dielectric constant, 


$$
G\left(k_{\alpha} r, k_{\alpha} r_{0}\right)=\left\{\begin{array}{l}
\frac{I_{0}\left(k_{\alpha} r_{0}\right)}{I_{0}\left(k_{\alpha} b\right)} \Delta_{0}\left(k_{\alpha} r, k_{\alpha} b\right), r>r_{0}, \\
\frac{I_{0}\left(k_{\alpha} r\right)}{I_{0}\left(k_{\alpha} b\right)} \Delta_{0}\left(k_{\alpha} r_{0}, k_{\alpha} b\right), r<r_{0},
\end{array}\right.
$$

$\Delta_{0}\left(k_{\alpha} r, k_{\alpha} b\right)=I_{0}\left(k_{\alpha} b\right) K_{0}\left(k_{\alpha} r\right)-I_{0}\left(k_{\alpha} r\right) K_{0}\left(k_{\alpha} b\right)$, $k_{\alpha}=k_{L i}$

Note that the above relation for dielectric constant (14) implies the well-known Liddein-Sachs-Teller relation $[5,6]$

$$
\frac{\omega_{L i}^{2}}{\omega_{T i}^{2}}=\frac{\varepsilon_{s t}}{\varepsilon_{o p t}},
$$

which determines the relationship between the frequencies of longitudinal and transverse phonons through the values of static and optical permittivities.

The integrand also has poles that are the roots of the equation

$$
D_{n}(\omega)=\frac{\omega^{2}}{c^{2}} \varepsilon(\omega)-\frac{\omega^{2}}{v_{0}^{2}}-\frac{\lambda_{n}^{2}}{b^{2}}=0 .
$$

Equation (17) determines the frequency spectrum of the radial harmonic with the number $n$ of electromagnetic waves excited by the REB in ion dielectric waveguide. With respect to the square of the frequency $\omega^{2}$, the spectrum equation (17) reduces to determining the roots of the quadratic equation. The frequencies $\omega_{l f(\mp)}$ corresponding to the roots of this equation lie in the low-frequency microwave and infrared ranges. The spectrum equation (17) can be written as follows

$$
\omega^{2}\left(\varepsilon_{\text {opt }} \frac{\left(\omega^{2}-\omega_{L i}^{2}\right)}{\left(\omega^{2}-\omega_{T i}^{2}\right)}-\frac{1}{\beta_{0}^{2}}\right)=\omega_{n}^{2},
$$

where $\beta_{0}=v_{0} / c, \omega_{n}=\lambda_{n} c / b$ is cutoff frequency of a vacuum waveguide of radius $b$. The roots of this equation are of the form

$$
\omega_{n l f(\mp)}^{2}=\frac{1}{2}\left(\omega_{n \Gamma}^{2} \mp \sqrt{\omega_{n \Gamma}^{4}-4 \omega_{n g}^{4}}\right) .
$$

Here

$$
\begin{gathered}
\omega_{n \Gamma}^{2}=\frac{1}{d_{o p t}}\left(\omega_{T i}^{2} d_{s t}+\omega_{n}^{2}\right), \omega_{n g}^{4}=\frac{1}{d_{o p t}} \omega_{T i}^{2} \omega_{n}^{2}, \\
d_{o p t}=\varepsilon_{o p t}-\beta_{0}^{-2}, d_{s t}=\varepsilon_{s t}-\beta_{0}^{-2} .
\end{gathered}
$$

For the frequency $\omega_{l f+}$ it is always $\omega_{l f+}>\omega_{L i}$, and for the frequency $\omega_{l f-}$ we have $\omega_{l f-}<\omega_{T i}$. In the most interesting limiting case

$$
\omega_{n}^{2}<<\omega_{T i}^{2}
$$

expressions for frequencies (19) are simplified

$$
\begin{gathered}
\omega_{n l f-}^{2}=\frac{\omega_{n}^{2}}{d_{s t}} \equiv \frac{\omega_{n}^{2} \beta_{0}^{2}}{\beta_{0}^{2} \varepsilon_{s t}-1}, \\
\omega_{n l f+}^{2}=\Omega_{T i}^{2}+\Delta \omega_{n}^{2}, \\
\Omega_{T i}^{2}=\omega_{T i}^{2} \frac{d_{s t}}{d_{o p t}}, \Delta \omega_{n}^{2}=\omega_{n}^{2} \frac{\Delta \varepsilon}{d_{s t} d_{o p t}}, \Delta \varepsilon=\varepsilon_{s t}-\varepsilon_{o p t} .
\end{gathered}
$$

Frequency $\omega_{n l f-}(21)$ is well known in the theory of wakefields excitation by REB in dielectric waveguides and resonators [1 - 3] and is in the microwave (terahertz) range. The frequency $\omega_{n l f+}$ lies in the infrared range and the process of wakefields excitation at this frequency, as it seems to us, has not been previously studied. For further analysis, the Fourier integral (11) is conveniently represented as

$$
S_{n}(\tau)=\frac{1}{d_{o p t}} \int_{-\infty}^{\infty} \frac{k_{\perp}^{2}(\omega) \omega}{k_{0}^{2} \varepsilon(\omega)} \frac{\left(\omega^{2}-\omega_{T i}\right) e^{-i \omega \tau}}{\left(\omega^{2}-\omega_{n l f-}^{2}\right)\left(\omega^{2}-\omega_{n l f+}^{2}\right)} d \omega .
$$

By calculating the residues in the poles $\omega= \pm \omega_{n l f-}-i 0, \quad \omega= \pm \omega_{n l f+}-i 0$, we find

$$
\begin{aligned}
S_{n}(\tau)= & -2 \pi i \frac{\lambda_{n}^{2}}{b^{2} d_{o p t}}\left(\frac{1}{\frac{\lambda_{n}^{2}}{b^{2}}+k_{n l f-}^{2}} \frac{\omega_{L i}^{2}-\omega_{n l f-}^{2}}{\omega_{n f+}^{2}-\omega_{n l f-}^{2}} \vartheta(\tau) \cos \omega_{n f f_{-}} \tau+\right. \\
& \left.+\frac{1}{\frac{\lambda_{n}^{2}}{b^{2}}+k_{n l f+}^{2}} \frac{\omega_{n l f+}^{2}-\omega_{L i}^{2}}{\omega_{n l f+}^{2}-\omega_{n l f-}^{2}} \vartheta(\tau) \cos \omega_{n f l+} \tau\right),
\end{aligned}
$$

where $k_{n l f(\mp)}=\omega_{n l f(\mp)} / v_{0}$. Accordingly, for the lowfrequency (infrared) part of the electromagnetic Green function, we obtain the following expression

$$
\begin{gathered}
E_{G z}^{(t)}(r, \tau)=d E_{z-}^{(t)}(r, \tau)+d E_{z+}^{(t)}(r, \tau), \\
d E_{z-}^{(t)}(r, \tau)=\frac{2 d Q}{d_{o p t}} \sum_{n=1}^{\infty} L_{n}^{(-)} \Pi_{n}\left(r, r_{0}\right) \vartheta(\tau) \cos \omega_{n l f-} \tau, \\
L_{n}^{(-)}=\frac{\lambda_{n}^{2}}{b^{2}\left(\frac{\lambda_{n}^{2}}{b^{2}}+k_{n l f-}^{2}\right)} \frac{\omega_{T i}^{2}-\omega_{n l f-}^{2}}{\omega_{n l f+}^{2}-\omega_{n l f-}^{2}}, \\
d E_{z+}^{(t)}(r, \tau)=\frac{2 d Q}{d_{o p t}} \sum_{n=1}^{\infty} L_{n}^{(+)} \Pi_{n}\left(r, r_{0}\right) \vartheta(\tau) \cos \omega_{n l f+} \tau, \\
L_{n}^{(+)}=\frac{\lambda_{n}^{2}}{b^{2}\left(\frac{\lambda_{n}^{2}}{b^{2}}+k_{n l f+}^{2}\right)} \frac{\omega_{n l f+}^{2}-\omega_{T i}^{2}}{\omega_{n l f+}^{2}-\omega_{n l f-}^{2}}, \\
\Pi_{n}\left(r, r_{0}\right)=\frac{J_{0}\left(\lambda_{n} r / b\right) J_{0}\left(\lambda_{n} r_{0} / b\right)}{N_{n}} .
\end{gathered}
$$

In the limiting case (20), the Green functions (23), (24) are simplified and take the form

$$
\begin{gathered}
d E_{z-}^{(t)}(r, \tau)=E_{w}^{(-)} \sum_{n=1}^{\infty} P_{n}\left(r, r_{0}\right) \vartheta(\tau) \cos \omega_{n l f-} \tau, \\
P_{n}\left(r, r_{0}\right)=\frac{J_{0}\left(\lambda_{n} r / b\right) J_{0}\left(\lambda_{n} r_{0} / b\right)}{J_{1}^{2}\left(\lambda_{n}\right)}, \\
d E_{z+}^{(t)}(r, \tau)=-E_{w}^{(+)} \vartheta(\tau) \cos \Omega_{T i} \tau \sum_{n=1}^{\infty}\left[\frac{1}{g} \frac{\Pi_{n}\left(r, r_{0}\right)}{\left(\frac{\lambda_{n}^{2}}{b^{2}}+k_{g}^{2}\right)}-\right. \\
\left.-\frac{1}{f} \frac{\Pi_{n}\left(r, r_{0}\right)}{\left(\frac{\lambda_{n}^{2}}{b^{2}}+k_{f}^{2}\right)}\right]
\end{gathered}
$$

where

$$
E_{w}^{(-)}=\frac{4 d Q}{b^{2} \varepsilon_{s t}}, \quad E_{w}^{(+)}=2 d Q \frac{\Omega_{T i}^{2}}{c^{2}} \frac{\beta_{0}^{2}}{d_{o p t}} \frac{\Delta \varepsilon}{\varepsilon_{s t}},
$$




$$
k_{g}^{2}=\frac{\Omega_{T i}^{2}}{c^{2} g}, k_{f}^{2}=\frac{\Omega_{T i}^{2}}{c^{2} f}, g=\frac{\Delta \varepsilon}{d_{s t} d_{o p t}}+\beta_{0}^{2}, f=\frac{\Delta \varepsilon}{d_{\text {opt }}}-1 \text {. }
$$

We neglected the dependence (22) of frequency $\omega_{n l f+}$ on the radial harmonic number, assuming that $\omega_{n l f+}=\Omega_{T i}$. In this case we can calculate the sums included in expression (26). As a result, for the Green function $d E_{z+}^{(t)}(r, \tau)$ we obtain the approximate expression

$$
\begin{gathered}
d E_{z+}^{(t)}(r, \tau)=E_{w}^{(+)} G_{T}\left(r, r_{0}\right) \vartheta(\tau) \cos \Omega_{T i} \tau, \\
G_{T}\left(r, r_{0}\right)=\frac{1}{f} G\left(k_{f} r, k_{f} r_{0}\right)-\frac{1}{g} G\left(k_{g} r, k_{g} r_{0}\right) .
\end{gathered}
$$

Expression (25) describes wake electromagnetic field excited by an infinitely thin electron ring bunch in ion dielectric waveguide in the microwave frequency range, in which there is no frequency dispersion of the dielectric constant. This expression for the wakefield coincides with that obtained in [1]. The exact expression (24) and approximate one (26) describe the excitation of wake electromagnetic waves belonging to a higherfrequency infrared branch of the electromagnetic waves of ion dielectric waveguide. In approximation (20), the frequencies of these waves (22) are practically independent on radial harmonic number. In this case, infrared electromagnetic radiation is excited much more efficiently than microwave radiation.

Thus, we obtained the Green function, which describes the longitudinal component of the wake electric field excited by a ring relativistic electron bunch in ion dielectric waveguide. The Green function contains the longitudinal (potential) and electromagnetic (vortex) parts. In the infrared range, the potential part is a field of longitudinal optical phonons. As for the electromagnetic part of the Green function, it contains a set of radial electromagnetic waves whose frequencies are in the microwave range, as well as electromagnetic radiation in the infrared frequency range.

\section{EXCITATION OF WAKEFIELDS BY AN ELECTRON BUNCH}

The resulting electromagnetic field $\vec{E}(r, \tau)$ of the electron bunch (4) can be found by summing the fields $\vec{E}_{G}$ of elementary electron ring charges (2).

We first consider the excitation of longitudinal optical phonons. Using the potential polarization part of the Green function (14), we obtain the following expression for the wakefield of longitudinal optical phonons

$$
E_{z}^{(l)}(r, \tau)=E_{L} \Gamma_{L}(r) Z\left(\omega_{L i} \tau\right),
$$

where

$$
\begin{aligned}
& Z(\omega \tau)=\frac{1}{t_{\text {eff }}} \int_{-\infty}^{\tau} T\left(\tau_{0} / t_{b}\right) \cos \omega\left(\tau-\tau_{0}\right) d \tau_{0}, \\
& \Gamma_{L}(r)=\frac{2 \pi}{s_{e f f}} \int_{0}^{b} R\left(r_{0} / r_{b}\right) G\left(k_{L i} r, k_{L i} r_{0}\right) r_{0} d r_{0}, \\
& E_{L}=2 Q \frac{\omega_{L i}^{2}}{v_{0}^{2} \varepsilon_{e f f}} .
\end{aligned}
$$

The function $Z(\omega \tau)$ describes the distribution of the wake field at a frequency $\omega$ in the longitudinal direction at each moment of time. We will consider an elec- tron bunch with a symmetric longitudinal profile $T\left(\tau_{0}\right)=T\left(-\tau_{0}\right)$. The wake function $Z(\omega \tau)$ is conveniently represented as

$$
Z(\omega \tau)=\frac{1}{\hat{\tau}}[\hat{T}(\Omega) \vartheta(\tau) \cos \omega \tau-X(\bar{\tau})],
$$

where $\Omega=\omega t_{b}, \bar{\tau}=\tau / t_{b}$,

$$
\begin{aligned}
X(\bar{\tau})= & \operatorname{sign} \tau \int_{|\bar{\tau}|}^{\infty} T(s) \cos \Omega(|\bar{\tau}|-s) d s, \\
& \hat{T}(\Omega)=2 \int_{0}^{\infty} T(s) \cos (\Omega s) d s, s=t / t_{b} .
\end{aligned}
$$

The first term (31) describes a wake wave propagating behind the bunch. The amplitude of the wake wave is equal to the Fourier amplitude (33) of the function that describes the longitudinal profile of the electron bunch. The second term in (31) describes the bipolar antisymmetric pulse of the polarization field, localized in the region of the bunch. The field of this pulse tends to zero with distance from the bunch.

Behind a bunch, the wake field (31) of longitudinal optical phonons has the form of a monochromatic wave

$$
E_{L i}(r, \tau)=E_{L} \Gamma_{L}(r) \frac{\widehat{T}\left(\Omega_{L i}\right)}{\hat{\tau}} \cos \omega_{L i} \tau, \quad \Omega_{L i}=\omega_{L i} t_{L} .
$$

We present the expressions for the Fourier amplitudes $\widehat{T}\left(\Omega_{L i}\right)$ for two model longitudinal profiles of the electron bunch: Gaussian and power laws

$$
\begin{aligned}
& T\left(\tau_{0} / t_{L}\right)=e^{-\tau_{0}^{2} / t_{L}^{2}}, \quad \hat{T}(\Omega)=\sqrt{\pi} e^{-\Omega^{2} / 4}, \\
& T\left(\tau_{0} / t_{L}\right)=\frac{1}{1+\tau_{0}^{2} / t_{L}^{2}}, \quad \hat{T}(\Omega)=\pi e^{-\Omega} .
\end{aligned}
$$

Longitudinal optical phonons are most efficiently radiated when the coherence condition is fulfilled $\omega_{L i} t_{L} \leq 1$. If the inequality $\omega_{L i} t_{L}>1$ takes place, then the longitudinal optical phonons are not coherently radiated and the amplitude of the wake wave is exponentially small.

Let's consider an electron bunch with a Gaussian transverse profile

$$
R(r)=e^{-r^{2} / r_{L}^{2}} .
$$

When the condition $k_{L i} b>>1$ is satisfied on the axis $r=0$ the function $\Gamma_{L}(r)$ takes on the value

$$
\begin{gathered}
\Gamma_{L}(0)=-\frac{1}{2} e^{\rho_{b}} \operatorname{Ei}\left(-\rho_{b}\right), \rho_{b}=\frac{k_{L i}^{2} r_{b}^{2}}{4}, \\
\operatorname{Ei}(z)=\int_{-\infty}^{z} \frac{e^{t}}{t} d t
\end{gathered}
$$

is integral exponential function. For thin $\rho_{b}<<1$ and wide $\rho_{b} \gg 1$ bunches the asymptotic representations for function (37) are

$$
\Gamma_{L}(0)= \begin{cases}\frac{1}{2} \ln \left(\frac{1}{\rho_{b}}\right), & \rho_{b}<<1, \\ \frac{1}{\rho_{b}}, & \rho_{b}>>1 .\end{cases}
$$

Thus, with the full coherence of the Cherenkov radiation of longitudinal optical phonons $\omega_{L i} t_{L} \leq 1$, $k_{L i} r_{b} \leq 1$ the wakefield of optical phonons on the axis of the waveguide takes the maximum value 


$$
\begin{aligned}
& \qquad E_{L i}(r, \tau)=E_{L} \ln \left(2 / k_{L i} r_{b}\right) \cos \omega_{L i} \tau . \\
& \text { where } E_{L i}(r, \tau)=2 Q \frac{\omega_{L i}^{2}}{v_{0}^{2} \varepsilon_{\text {eff }}} .
\end{aligned}
$$

We present the expressions for the amplitude of the wakefield on the axis of the waveguide $E_{L}$ for two ion dielectrics of the alkali-halogen group: sodium chloride $\mathrm{NaCl}$ and potassium iodide KI. For sodium chloride, we have

$$
E_{L}=0.2 N_{0}(\mathrm{~V} / \mathrm{cm}) .
$$

Here $N_{0}=Q / e$ is the number of electrons in the bunch. The frequency of longitudinal optical phonons is equal $f_{L i}=7.62 \cdot 10^{12} \mathrm{~Hz}$. For $N_{0}=10^{11}$ from this formula we obtain the estimation for the electric field strength $E_{L}=2 \mathrm{GV} / \mathrm{cm}$. Accordingly, for potassium iodide we have

$$
E_{L}=0.11 N_{0}(\mathrm{~V} / \mathrm{sm})
$$

The frequency of longitudinal optical phonons and the electric field strength are equal $f_{T i}=4 \cdot 10^{12} \mathrm{~Hz}$, $E_{L}=1.1 \mathrm{GV} / \mathrm{cm}$, lower than in the previous case.

Let us now consider the excitation of wake electromagnetic waves by an electron bunch. Using the electromagnetic Green function, we obtain the wake electromagnetic field as a superposition of radial modes

$$
\begin{aligned}
& E_{z(\mp)}^{(t)}(r, \tau)=4 \frac{Q}{b^{2} d_{o p t}} E_{0} \sum_{n=1}^{\infty} L_{n}^{(\mp)} \Gamma_{n} \frac{J_{0}\left(\lambda_{n} r / b\right)}{J_{1}^{2}\left(\lambda_{n}\right)} Z_{n}^{(\mp)}(\tau),(39) \\
& \Gamma_{n}=\frac{2 \pi}{S_{\text {eff }}} \int_{0}^{b} R\left(\frac{r_{0}}{r_{b}}\right) J_{0}\left(\lambda_{n} \frac{r}{b}\right) r_{0} d r_{0}, Z_{n}^{(\mp)}(\tau)=Z\left(\omega_{n f(\mp)} \tau\right) .
\end{aligned}
$$

For a symmetric electron bunch in the "wave zone" $\omega_{n f(\mp)} \tau>>1$, where the quasistatic field of the electron bunch is small, the wake field (39) is a superposition of radial modes of the dielectric waveguide

$$
E_{z(\mp)}^{(t)}(r, \tau)=E_{0} \sum_{n=1}^{\infty} L_{n}^{(\mp)} \Gamma_{n} \frac{\hat{T}_{n}^{(\mp)}}{\hat{\tau}} \frac{J_{0}\left(\lambda_{n} r / b\right)}{J_{1}^{2}\left(\lambda_{n}\right)} \cos \omega_{n l f(\mp)} \tau,
$$

where $E_{0}=4 \frac{Q}{b^{2} d_{\text {opt }}}, \hat{T}_{n}^{(\mp)} \equiv \hat{T}\left(\Omega_{n(\mp)}\right)$ is Fourier component (35) of the function $T(s)$ at the dimensionless frequency $\Omega_{n(\mp)}=\omega_{n l(\mp)} t_{b}$. For an electron bunch with a Gaussian longitudinal (35) and transverse (36) profiles, the coefficients $\Gamma_{n}$ and $\hat{T}_{n}^{(\mp)}$, which are determined by the specific form of the transverse and longitudinal density profiles of the bunch, have the form

$$
\begin{aligned}
& \hat{T}_{n}^{(\mp)}=\exp \left(-\frac{1}{4} \Omega_{n(\mp)}^{2}\right), \Gamma_{n}=2 \int_{0}^{1 / \eta_{b}} J_{0}\left(\lambda_{n} \eta_{b} \rho\right) e^{-\rho^{2}} \rho d \rho, \\
& \eta_{b}=r_{b} / b .
\end{aligned}
$$

When the condition $\eta_{b}<<1$ is satisfied, the expression for the coefficient $\Gamma_{n}$ is simplified

$$
\Gamma_{n}=\exp \left(-\frac{1}{4} \lambda_{n}^{2} \eta_{b}^{2}\right)
$$

If condition (20) is satisfied, we can use approximate expressions for the Green functions (25), (26). As a result, for wakefields excited by electron bunches with Gaussian longitudinal and transverse profiles, instead of exact expressions (39), we obtain approximate relations

$$
\begin{gathered}
E_{z-}^{(t)}(r, \tau)=\frac{4 Q}{b^{2} \varepsilon_{s t}} \sum_{n=1}^{\infty} e^{-\frac{1}{4}\left(\Omega_{n-+}^{2} \lambda_{n}^{2} \eta_{b}^{2}\right)} \frac{J_{0}\left(\lambda_{n} \frac{r}{b}\right)}{J_{1}^{2}\left(\lambda_{n}\right)} \cos \omega_{n l f-} \tau, \\
E_{z+}^{(t)}(r, \tau)=2 Q \frac{\Omega_{T i}^{2}}{c^{2}} \frac{\Delta \varepsilon}{\left(\varepsilon_{o p t}-1\right) \varepsilon_{s t}} e^{-\frac{\Omega_{T h}^{2} t_{n}^{2}}{4}} \Gamma_{T}(r) \cos \Omega_{T i} \tau, \\
\Gamma_{T}(r)=\frac{2 \pi}{s_{e f f}} \int_{0}^{b} e^{-\frac{r_{0}^{2}}{r_{b}^{2}}} G_{T}\left(r, r_{0}\right) r_{0} d r_{0} .
\end{gathered}
$$

If $k_{f, g} b>>1$ on the axis $r=0$ the function $\Gamma_{T}(r)$ takes the value

$$
\Gamma_{T}(0)=-\frac{1}{2}\left(\frac{1}{f} e^{\rho_{f}} E i\left(-\rho_{f}\right)-\frac{1}{g} e^{\rho_{g}} E i\left(-\rho_{g}\right)\right),
$$

$\rho_{f, g}=\frac{k_{f, g}^{2} r_{b}^{2}}{4}$. When $\rho_{f, g}<<1$ we have instead of $(43)$

$$
\Gamma_{T}(0)=\frac{1}{f} \ln \left(\frac{2}{k_{f} b}\right)-\frac{1}{g} \ln \left(\frac{2}{k_{g} b}\right) .
$$

From expression (41) it follows that an electron bunch excites finite number of radial modes of microwave radiation, for which the coherence condition $\omega_{n l f-}^{2} t_{b}^{2} \leq 1, \lambda_{n}^{2} r_{b}^{2} \leq 1$ for excitation by an electron bunch is satisfied. Since the condition $\Omega_{T i}^{2} b^{2} / c^{2}>>1$ as rule is fulfilled, then the REB will primarily excite infrared radiation (42) upon its coherent excitation $\Omega_{T_{i}}^{2} t_{b}^{2} \leq 1$.

\section{CONCLUSIONS}

In this work, the process of the excitation of Cherenkov wake electromagnetic waves by a relativistic electron bunch in ion dielectric waveguide is studied. The ion dielectrics of the alkali-halide group are considered. In ion dielectrics of this group in the infrared and microwave frequency ranges, there are three branches of electromagnetic waves. Two of them correspond to transverse electromagnetic waves. In the infrared range there is also a branch corresponding to the optical longitudinal phonons of an ion dielectric. For all these branches, analytical expressions for the wake electromagnetic field excited by a relativistic electron bunch were obtained and studied. The frequency spectrum and the spatio-temporal structure of the Cherenkov wakefield are determined. It is shown that in the infrared (microwave) frequency range the excited wake electric field consists of potential field of longitudinal optical phonons and a set of eigen electromagnetic waves of a dielectric waveguide.

The frequency spectrum and the spatio-temporal structure of the Cherenkov wake field are determined. It is shown that in the infrared (microwave) frequency range the excited electric field of the wakewaves consists of potential field of longitudinal optical phonons and the set of eigen electromagnetic waves of an ion dielectric waveguide.

\section{REFERENCES}

1. V.A. Balakirev, N.I. Karbushev A.O. Ostrovsky, Yu.V. Tkach. Theory of Cherenkov amplifiers and generators based on relativistic beams. Kiev. "Naukova Dumka”, 1993, 207 p. 
2. V.A. Balakirev, I.N. Onishchenko, D.Yu. Sidorenko, G.V. Sotnikov. Chardge particle accelerated by wakefields in a dielectric resonator with exciting electron bunch channel // Technical Physics Letters. 2003, v. 29, № 7, p. 589-591.

3. V.I. Maslov, I.N. Onishchenko. Transformation ratio at wakefield excitation in dielectric resonator by shaped sequence of electron bunches with linear growth of current // Problems of Atomic Science and Technology. Series "Plasma Electronics and New Methods of Acceleration”. 2013, № 4, p. 69-72.
4. V.A. Balakirev, I.N. Onishchenko. Cherenkov radiation of a laser pulse in ion dielectrics // Problems of Atomic Science and Technology. Series "Plasma Electronics and New Methods of Acceleration”. 2019, № 4, p. 39-47.

5. M. Bourne, H. Coon. The dynamic theory of the crystal lattice. M.: "Foreign literature", 1958, 488 p.

6. J. Slater. Dielectrics, semiconductors, metals. M.: "Mir", 1969, 664 p.

7. N. Ashkfort, N. Merlin. Solid State Physics. M.: "Mir", 1978, v. 2, 392 p.

Article received 22.10.2019

\section{ЧЕРЕНКОВСКОЕ ИЗЛУЧЕНИЕ РЕЛЯТИВИСТСКОГО ЭЛЕКТРОННОГО СГУСТКА В ИОННОЙ ДИЭЛЕКТРИЧЕСКОЙ СРЕДЕ}

\section{В.А. Балакирев, И.Н. Онищенко}

Исследован процесс возбуждения Черенковского электромагнитного излучения релятивистским электронным сгустком в ионном диэлектрическом волноводе. Получена и исследована пространственновременная структура кильватерного поля в ионном диэлектрическом волноводе. Показано, что возбуждаемое кильватерное поле в инфракрасном диапазоне и больших длинах волн состоит из поля продольных оптических фононов и набора собственных электромагнитных волн диэлектрического волновода.

\section{ЧЕРЕНКІВСЬКЕ ВИПРОМІНЮВАННЯ РЕЛЯТИВІСТСЬКОГО ЕЛЕКТРОННОГО ЗГУСТКА В ІОННОМУ ДІЕЛЕКТРИЧНОМУ СЕРЕДОВИЩІ}

\section{В.А. Балакірєв, І.М. Оніщенко}

Досліджено процес збудження Черенківського електромагнітного випромінювання релятивістським електронним згустком в іонному діелектричному хвилеводі. Отримана і досліджена просторово-часова структура кільватерного поля в іонному діелектричному хвилеводі. Показано, що збуджене кільватерне поле в інфрачервоному діапазоні і більших довжинах хвиль складається з поля поздовжніх оптичних фононів і набору власних електромагнітних хвиль діелектричного хвилеводу. 